\title{
Influence of Internal Fixator Stiffness on Murine Fracture Healing: Two Types of Fracture Healing Lead to Two Distinct Cellular Events and FGF-2 Expressions
}

\author{
Masaki UENO ${ }^{1)}$, Ken URABE ${ }^{1)}$, Kouji NARUSE ${ }^{1)}$, Kentaroo UCHIDA ${ }^{1)}$, \\ Hiroaki MINEHARA ${ }^{1)}$, Takeaki YAMAMOTO ${ }^{1)}$, Roland STECK ${ }^{2)}$, Laura GREGORY ${ }^{2)}$, \\ Martin E. WULLSCHLEGER ${ }^{2,3)}$, Michael A. SCHUETZ ${ }^{2,3)}$, and Moritoshi ITOMAN ${ }^{4}$ \\ ${ }^{1)}$ Department of Orthopaedic Surgery, Kitasato University School of Medicine, 1-15-1 Kitasato, Sagamihara, \\ Kanagawa 228-8555 Japan, ${ }^{2}$ Institute of Health and Biomedical Innovation, Queensland University \\ of Technology, 60 Musk Avenue, Kelvin Grove Urban Village, Kelvin Grove, Queensland 4059, \\ ${ }^{3)}$ Trauma Services, The Princess Alexandra Hospital, 199 Ipswich Road Woolloongabba, Brisbane, \\ Queensland 4102, Australia, ${ }^{4}$ Department of Orthopaedic Surgery, Kyushu Rosai Hospital, \\ 1-3-1 Kuzuharatakamastu, Kokuraminami-ku, Kitakyushu, Fukuoka 800-0296, Japan
}

\begin{abstract}
This study aimed to clarify the relationship between the mechanical environment at the fracture site and endogenous fibroblast growth factor-2 (FGF-2). We compared two types of fracture healing with different callus formations and cellular events using MouseFix ${ }^{\mathrm{TM}}$ plate fixation systems for murine fracture models. Left femoral fractures were induced in 72 ten-week-old mice and then fixed with a flexible (Group F) or rigid (Group R) Mouse Fix ${ }^{\mathrm{TM}}$ plate. Mice were sacrificed on days $3,5,7,10,14$, and 21 . The callus volumes were measured by 3D micro-CT and tissues were histologically stained with hematoxylin \& eosin or safranin-O. Sections from days 3, 5, and 7 were immunostained for FGF-2 and Proliferating Cell Nuclear Antigen (PCNA). The callus in Group F was significantly larger than that in Group R. The rigid plate allowed bone union without a marked external callus or chondrogenesis. The flexible plate formed a large external callus as a result of endochondral ossification. Fibroblastic cells in the granulation tissue on days 5 and 7 in Group F showed marked FGF2 expression compared with Group R. Fibroblastic cells showed ongoing proliferation in granulation tissue in group F, as indicated by PCNA expression, which explained the relative granulation tissue increase in group $F$. There were major differences in early phase endogenous FGF-2 expression between these two fracture healing processes, due to different mechanical environments.
\end{abstract}

Key words: callus formation, fracture, growth factor, internal fixator, mouse 


\section{Introduction}

Fracture healing processes are influenced by the mechanical environment at the fracture site. Fixation properties between bone fragments affect morphological characteristics $[6,8]$, and callus volume is high in a dynamic environment where the fracture site is unstable and slight strain exists [24]. However, previous studies on the relationship between fixation stability and fracture repair processes have been limited to showing morphological changes involving the fracture callus, and the precise mechanism has yet to be fully elucidated.

Recent studies of fracture healing have shown that growth factors are involved in regulating cell proliferation, differentiation and matrix synthesis. However, fracture healing processes are not all the same. Various mechanical environments at the fracture site may result in different expressions of endogenous growth factors.

Previous studies have shown that the production of fibroblast growth factors (FGFs) at the fracture site plays an important role in fracture repair processes, that the effects of FGFs are exerted mainly on mesenchymal cell proliferation, and that FGF-2 is a more potent mitogen for fibroblasts and pre-osteoblasts than for differentiated osteoblasts [20]. The local application of recombinant human FGF-2 has also been shown to accelerate fracture healing through the formation of a large external callus in the cortical bone at an early stage [14, 19, 21, 22, 27]. Therefore, we speculated that FGF-2 is among the factors most strongly influencing callus size as determined by the mechanical environment.

To clarify the relationship between the mechanical environment at the fracture site and endogenous FGF-2, we observed two types of fracture healing with distinct callus formation and cellular events using MouseFix ${ }^{\mathrm{TM}}$ plate fixation systems for murine fracture models. Furthermore, we compared endogenous growth factor expressions during fracture healing processes under controlled experimental conditions.

\section{Materials and Methods}

Mouse Fix ${ }^{\mathrm{TM}}$ is a locking plate system with good angular stability that includes two plate types: a rigid (Fig. 1A) and a flexible (Fig. 1B) plate [10, 18]. The rigid

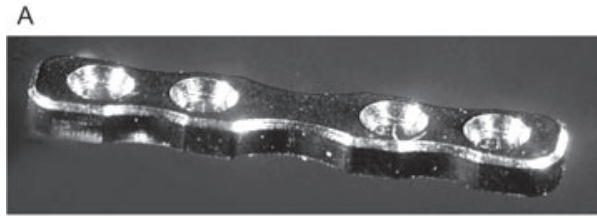

B

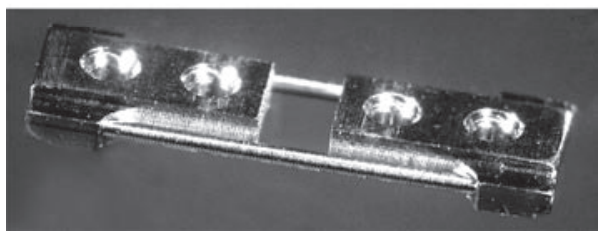

Fig. 1. Mouse Fix ${ }^{\mathrm{TM}}$. (A) Rigid plate, (B) Flexible plate. There are 4 holes for screw thread cutting. The flexible plate is comprised of two segments bridged by wires which are $0.25 \mathrm{~mm}$ in diameter. Each plate is fixed with locking screws.

plate is $7.75 \times 1.5 \mathrm{~mm}$ and the flexible plate is $7.75 \times$ $2.5 \mathrm{~mm}$ (both are $0.7 \mathrm{~mm}$ in thickness). The diameter of the bridging wire of the flexible plate is $0.25 \mathrm{~mm}$. Each plate has 4 holes for screw thread cutting. The screws are $2 \mathrm{~mm}$ in length, the outside diameter of the thread is $0.5 \mathrm{~mm}$, and the core diameter is $0.36 \mathrm{~mm}$. The head of each screw also allows for screw thread cutting.

Ten-week-old male C57BL/6J mice $(\mathrm{n}=72$; mean weight, $24.5 \mathrm{~g}$; Charles River Laboratories Japan, Inc., Yokohama, Japan) were used as experimental models. Mice were allowed to adjust to the new environment for 2 weeks and were randomly allocated between 2 experimental groups, Group R (fixed with rigid plate) and Group F (fixed with flexible plate), and 6 mice were sacrificed on each scheduled day of sacrifice. Each cage $(225 \times 338 \times 140 \mathrm{~mm})$ housed 4 mice. Mice were fed an ordinary laboratory diet (CRF-1, Oriental Yeast Co., Ltd., Tokyo, Japan) and given access to an automatic water dispenser containing water treated with UV irradiation and filtered through $5-\mu \mathrm{m}$ pores. The rearing conditions were as follows: SPF grade; temperature, 25 $\pm 2^{\circ} \mathrm{C}$ in summer and $23 \pm 2^{\circ} \mathrm{C}$ in winter; relative humidity, $55 \pm 10 \%$; ventilation count, $\geq 10$ times $/ \mathrm{h}$; cleanliness class, 10,000 at $0.5 \mu \mathrm{m}$, wet circulation; noise, $\leq 60 \mathrm{~dB}$; luminance, $300 \mathrm{~lx}$; light/dark cycle, $12 \mathrm{~h} / 12 \mathrm{~h}$.

All surgeries and handling procedures were in accor- 
dance with the terms of the Animal Ethics Committee of Kitasato University.

Mice were initially sedated using diethyl ether, and a mixture of 1 part midazolam, 3 parts Domitor, and 1 part Vetorphale was then intramuscularly injected into the upper limbs. Based on an optimal dose of $0.05 \mathrm{ml} / 100$ $\mathrm{g}, 0.02 \mathrm{ml}$ of this anesthetic was administered. The operative procedure was applied only to the left femur. A 1-cm lengthwise incision was made between the hip and the knee. The fascia was cut and the muscle was retracted, taking care to avoid injury to the periosteum. The plate guide instrument with the plate was positioned on the femur. The second hole from the proximal end was drilled first, and the screw was placed and then twisted off. Next, the third hole from the proximal end was drilled, and the screw was placed. Screws were inserted into the other two holes, keeping the pins attached to the screws to stabilize the instrument for sawing. The bone was gently cut using a wire saw (diameter, $0.22 \mathrm{~mm}$ ) to create a gap. Finally, the last pin was twisted off. After surgery, irrigation with saline was performed to remove small bone fragments. Success of the surgical procedure was confirmed radiographically. The distance between the plate and the bone also affects the dynamic environment of the fracture sites. Therefore, we measured the space between both ends of the plate and the bone. Distances were measured using the "Scion Image 4.0" image analysis software (Scion Co., Ltd., Frederick, MD, USA).

After surgery, the mice were allowed to use their fractured legs without restriction. In order to assess chronological changes in healing, 6 mice in each group were sacrificed with excess $\mathrm{CO}_{2}$ gas at $3,5,7,10,14$, and 21 days after fracture induction.

\section{External hard callus volume}

Femurs were excised with the surrounding muscle and the implants were carefully removed. The femurs were placed in $4 \%$ paraformaldehyde, and stored in a refrigerator $\left(4^{\circ} \mathrm{C}\right)$ for $48 \mathrm{~h}$. The $4 \%$ paraformaldehyde was then replaced with phosphate-buffered saline (PBS). Micro-CT images were obtained using a micro focus X-ray CT system (inspeXio SMX-90CT: Shimadzu Co., Ltd., Tokyo, Japan). Images were obtained employing the following settings: acceleration voltage, $90 \mathrm{kV}$; cur- rent, $110 \mu \mathrm{A}$; voxel size, $57 \mu \mathrm{m} /$ pixel; matrix size, 512 $\times 512$. The external hard callus volume was measured three-dimensionally using VGStudioMAX 2.0 (Nihon Visual Science, Inc., Tokyo, Japan) with the volumerendering method. The threshold of each image was kept at the same level using the software function.

\section{Histological evaluation}

After micro-CT scanning, the femur was demineralized with a $10 \%$ EDTA solution for 10 days. The remaining tissue was embedded in paraffin. Subsequently, $4-\mu \mathrm{m}$ sections were cut through the long axis of each femur in the coronal plane. The section with the maximum diameter was chosen as being representative of each fracture. All sections were then subjected to hematoxylin \& eosin (HE) and safranin-O staining. To evaluate the cellular event progression, the number of samples in which pathognomonic cellular events appeared was compiled for each group according to the sacrifice schedule.

Sections from days 3, 5, and 7 were immunolabeled with anti-FGF-2 rabbit polyclonal antibody (Santa Cruz Biotechnology; Santa Cruz, CA, USA) and with antiPCNA (Proliferating Cell Nuclear Antigen) rabbit polyclonal antibody (Santa Cruz Biotechnology). After labeling, sections were stained with the streptavidinbiotin-peroxidase system (HISTOFINE SAB-PO kit, Nichirei, Tokyo, Japan) using 3.3-diaminobenzidine (DAB). All immunostained sections were counterstained with Meyer's hematoxylin.

\section{Statistical analysis}

Statistical evaluation was performed with SPSS software, version 12.0 (SPSS Inc., Chicago, IL, USA). Student's $t$-tests were conducted to determine differences between groups and values of $P<0.05$ were considered significant.

\section{Results}

\section{External hard callus volume}

The average bone-plate gap at both ends of the plate was $0.01 \pm 0.02 \mathrm{~mm}$ (range, $0-0.08 \mathrm{~mm}$ ) in Groups $\mathrm{R}$ and F; thus, plate floating was practically absent. As shown in Fig. 2, radiographs demonstrated that we were 

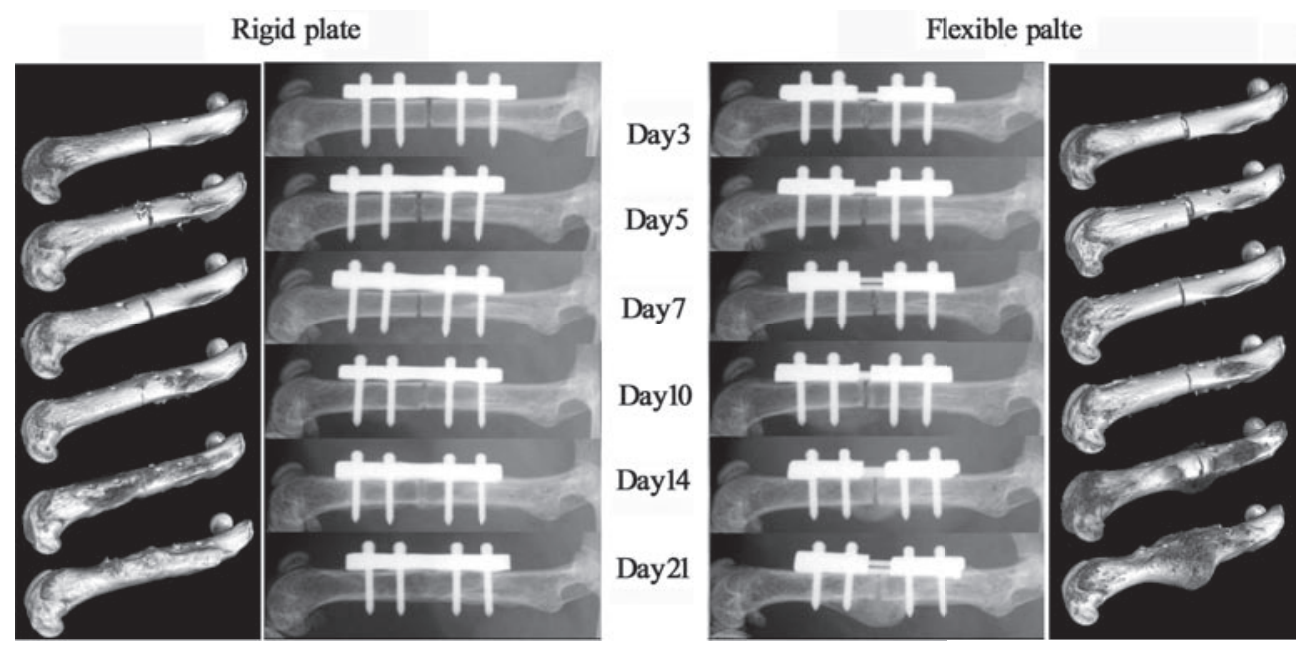

Fig. 2. Radiographs and $3 \mathrm{D}$ micro-CT analysis. Representative soft $\mathrm{X}$-ray images and $3 \mathrm{D}$ micro-CT analysis images of Group R and Group F specimens at each time point after fracture. As the bones approached union, a large external callus was observed at the fracture site in Group F. In contrast, almost no external callus was observed in Group R.

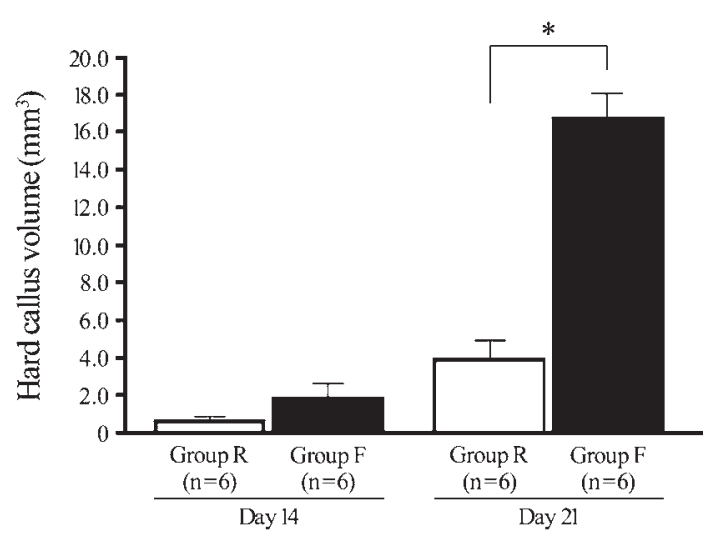

Fig. 3. Analysis of external hard callus volume using 3D micro-CT analysis data on days 14 and 21. Data are given as means $\pm \mathrm{SD} ; * P<0.05$.

able to position the plate ideally. A large amount of external callus was observed at the fracture site in Group F. In contrast, almost no external callus was observed in Group R (Fig. 2). Mineralized callus was not detected on days 3 to 10 ; however, the external hard callus volume began to increase from day 14 in both groups. On day 21, a marked increase was observed in both groups, especially Group F, in which the volume was significantly greater than that in Group $\mathrm{R}(P<0.05)$ (Fig. 3).

\section{Histomorphometry}

On day 3 in Group R, inflammatory cell infiltration was localized adjacent to the fractured bone ends (Fig. 4A). In Group F, inflammatory cell infiltration and hematoma formation along the fracture line were evident on the medullary side (Fig. 5A). In Group F, the cells in the inner layer of the periosteum proliferated and crossed the fracture site on day 5 (Fig. 5B). However, in Group $\mathrm{R}$, the proliferation of cells in the inner layer of the periosteum was minimal. Fibrous cells were numerous in the fracture gap and were aligned parallel to the fracture line (Fig. 4B). On day 7, in Group R, subperiosteal cell proliferation and intramembranous ossification occurred under the periosteum. Newly formed woven bone was present in the fracture gap, and a small intramedullary callus containing predominantly fibrous tissue appeared in the medullary cavity (Fig. 4C). In Group F, while subperiosteal cell proliferation and intramembranous ossification occurred under the periosteum, the cells adjacent to the newly formed trabecular bone increased in size and synthesized an avascular matrix, forming cartilage (Fig. 5C, arrowheads). An intramedullary callus containing predominantly fibrous tissue and a small area of newly formed woven bones developed around the fracture site. On day 10, the fracture gap was filled with newly formed woven bone in Group R and no chondro- 

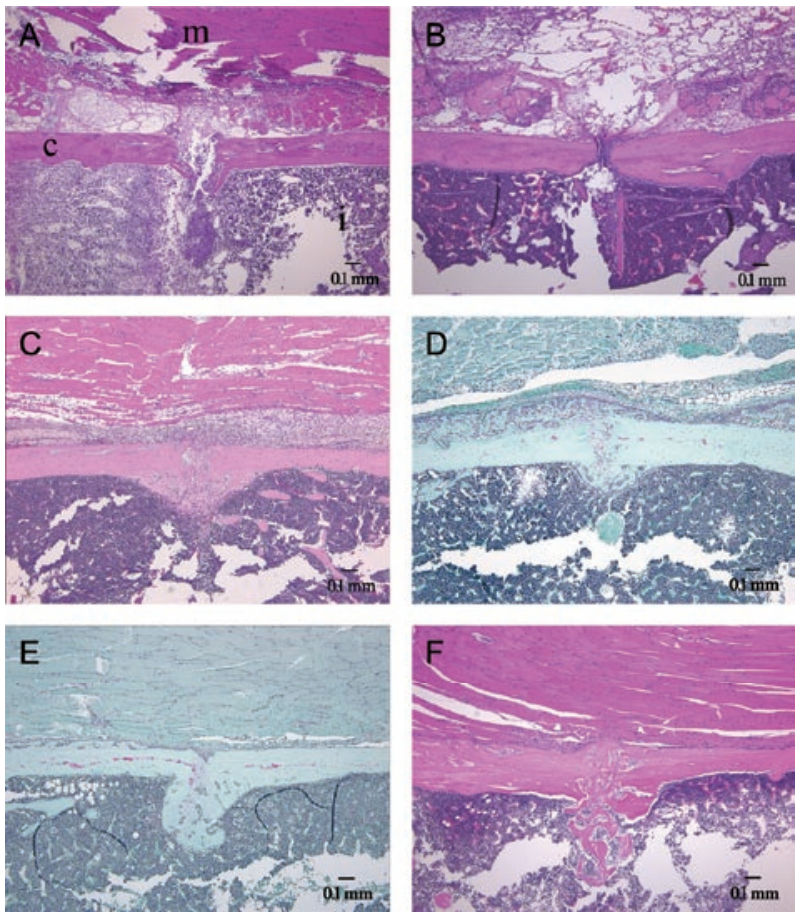

Fig. 4. Tissue samples after hematoxylin and eosin staining on days $3,5,7$, and 21 , and safranin-O staining on days 10 and 14 in Group R. Days (A) 3, (B) 5, (C) 7, (D) 10, (E) 14 , and $(\mathrm{F}) 21$. Specific regions are labeled as follows: cortical bone, c; muscle, m; intramedullary canal, i.

genesis was observed in the external callus. The small intramedullary callus also consisted of newly formed woven bone (Fig. 4D). Meanwhile, in Group F, cartilage containing mainly proliferating and hypertrophic chondrocytes was the predominant tissue in the external callus. In the intramedullary callus, newly formed woven bone was also the predominant tissue (Fig. 5D).

On day 14 , in Group R, a new lamellar bone layer had formed under the periosteum. Remodeling of the woven bone progressed in the fracture gap and the intramedullary callus on day 14 (Fig. 4E). Formation of a medullary cavity in the woven bone of the fracture gap and resorption of the intramedullary callus were observed on day 21 (Fig. 4F).

On day 14, in Group F, endochondral ossification was present from the surrounding trabecular bone to the center of the external callus. In the intramedullary callus, remodeling of the woven bone progressed and bony unions appeared in the medullary cavity (Fig. 5E). On
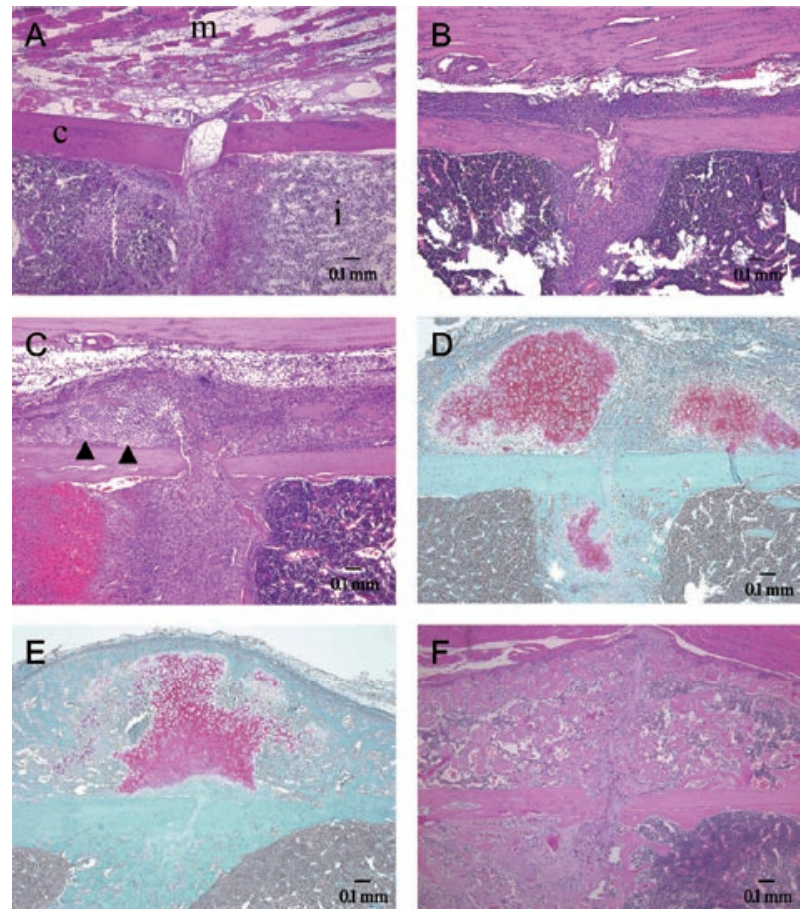

Fig. 5. Hematoxylin and eosin stained tissue samples collected on days $3,5,7$ and 21 , and safranin-O stained samples collected on days 10 and 14 in Group F. Days (A) 3, (B) 5, (C) 7, (D) 10, (E) 14, and (F) 21. Specific regions are labeled as follows: cortical bone, c; muscle, $\mathrm{m}$; intramedullary canal, i.

day 21 , chondrocytes disappeared, newly formed woven bone was filled, and bony union was achieved in the external callus. Resorption of the new trabecular bone and formation of a medullary cavity occurred in the external callus. A medullary cavity had formed in the original cortical bone, and reestablishment of new cortical bone had also progressed (Fig. 5F).

\section{Cellular event progression in group $F$ and group $R$}

"Intramedullary callus formation" was observed in 1 of 6 mice on day 5 and in all animals on day 7 in Group R (Table 1). Table 2 shows that each cellular event, including "proliferation of periosteum", "chondrogenesis", "endochondral ossification", and "hard callus bridging" appeared on the same day in the majority of mice in Group F.

\section{Immunohistochemistry}

Figure 6A and 6B show minimal FGF-2 staining of the periosteum on day 3 in both groups. Few PCNA- 
Table 1. Number of fractures with each cellular event after fracture in Group $\mathrm{R}$

\begin{tabular}{lccc}
\hline \multicolumn{1}{c}{ Group R } & Day 3 & Day 5 & Day 7 \\
\hline Infiltration of fibrous tissue into the fracture gap & - & $6 / 6$ & - \\
Intramedullary callus formation & - & $1 / 6$ & $6 / 6$ \\
\hline
\end{tabular}

Data indicate the number of fractures with each cellular event per total number of fractures.

Table 2. Number of fractures with each cellular event after fracture in Group F

\begin{tabular}{lcccccc}
\hline \multicolumn{1}{c}{ Group F } & Day 3 & Day 5 & Day 7 & Day 10 & Day 14 & Day 21 \\
\hline Proliferation of periosteum & $1 / 6$ & $6 / 6$ & - & - & - & - \\
Chondrogenesis & - & - & $6 / 6$ & - & - & - \\
Endochondral ossification & - & - & - & $1 / 6$ & $6 / 6$ & - \\
Hard callus bridging & - & - & - & - & - & $6 / 6$ \\
\hline
\end{tabular}

Data indicate the number of fractures with each cellular event per total number of fractures.
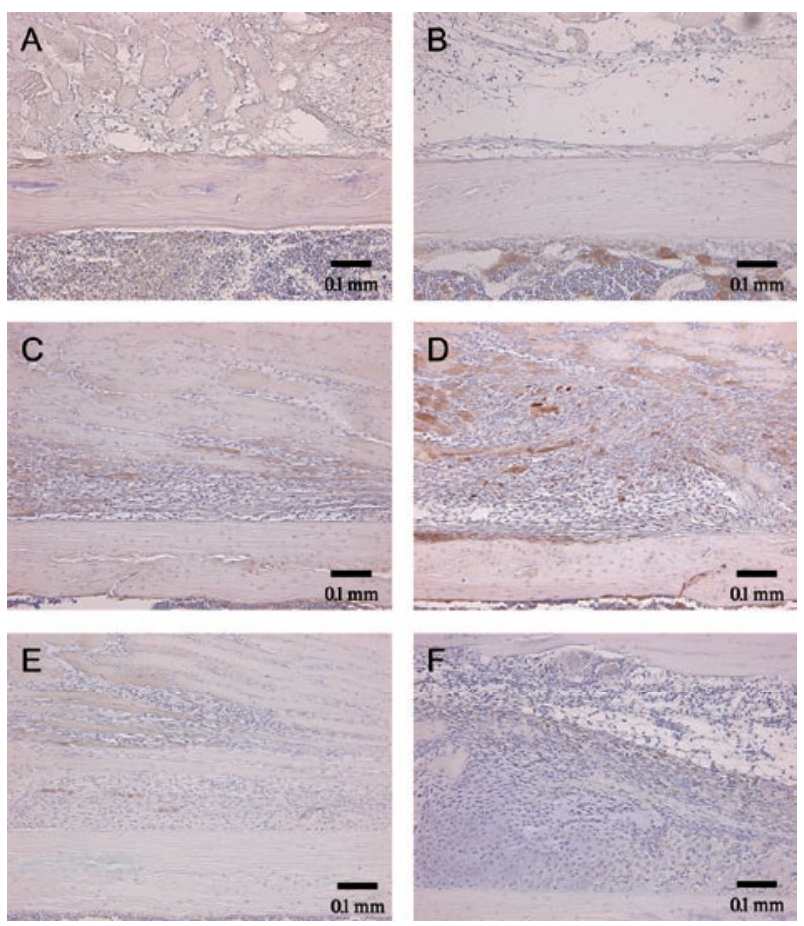

Fig. 6. Immunohistochemical localization of FGF-2 on days 3,5, and 7. (A) on day 3 in Group R, (B) on day 3 in Group F, (C) on day 5 in Group R, (D) on day 5 in Group F, (E) on day 7 in Group R, (F) on day 7 in Group F.

positive cells were detected in the same area of Group F (Fig. 7B). In Group F, FGF-2 was widely distributed in the proliferated granulation tissue and thickened periosteum on day 5 (Fig. 6D). In Group R, relatively little immunostaining was observed in these areas (Fig.
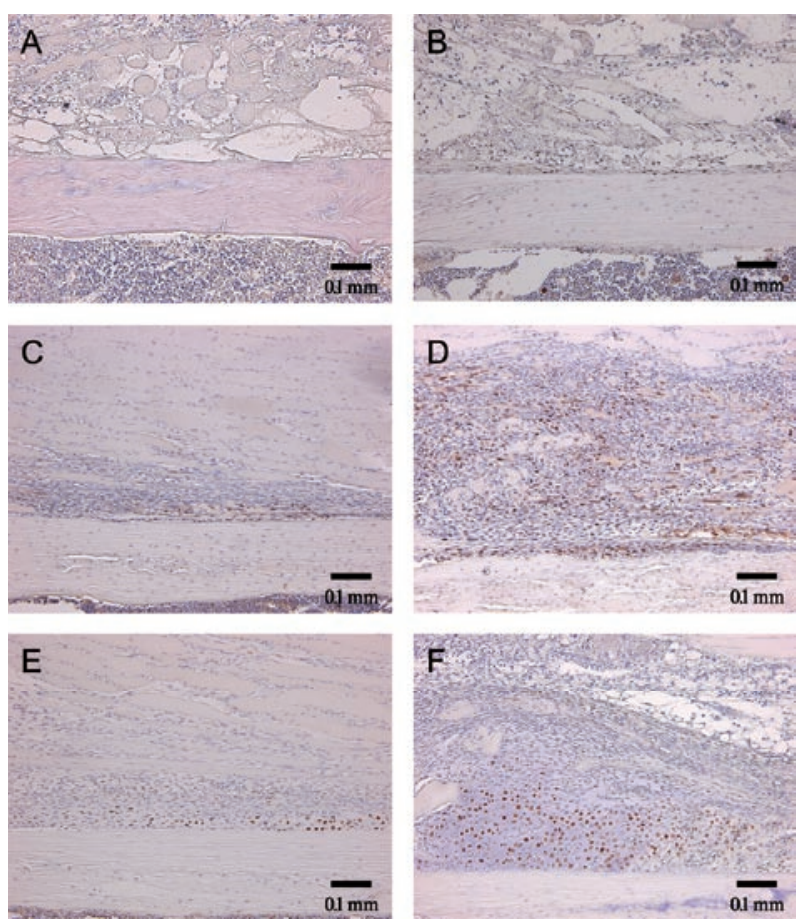

Fig. 7. Immunohistochemical localization of PCNA on days 3,5, and 7. (A) on day 3 in Group R, (B) on day 3 in Group F, (C) on day 5 in Group R, (D) on day 5 in Group F, (E) on day 7 in Group R, (F) on day 7 in Group F.

6C). In group F, many PCNA-positive cells were detected in the periosteum on day 5 (Fig. 7D), whereas few cells under the periosteum were stained with PCNA in Group R (Fig. 7C). Fibroblastic cells in granulation tissue were PCNA-positive in both groups on day 5 (Fig. 
7C and 7D), and some staining persisted on day 7 only in Group F (Fig. 7F). In Group F on day 7, as shown in Fig. 6F, cells, including immature chondrocytes and mesenchymal cells under the periosteum, were specifically immunostained by anti-FGF-2 antiserum. Proliferating chondrocytes and pre-hypertrophic chondrocytes were PCNA-positive (Fig. 7F). In contrast, only a few PCNA-positive cells above the cortex were detected in Group R on day 7 (Fig. 7E).

\section{Discussion}

The present results confirmed the external callus to be significantly larger in Group F than in Group R. Fibroblastic cells in granulation tissue on days 5 and 7 in Group F demonstrated marked FGF-2 expression. The granulation tissue area was already slightly larger in Group $\mathrm{F}$ than in Group R by day 5, but ongoing cell proliferation in granulation tissue, as indicated by PCNA expression, explained the relative granulation tissue increase in group $\mathrm{F}$.

The fracture healing process is reportedly influenced by the mechanical environment at the fracture site $[6,8$, 24]. In the present study, we observed cellular events at the fracture healing site using two MouseFix ${ }^{\mathrm{TM}}$ plates with different levels of flexibility. Our histological analysis showed proliferation of cells in the inner layer of the periosteum to be seen first, by day 5, in Group R with the rigid plate. External callus formation via intramembranous ossification was slight 7 days after the fracture. The fracture gap was filled with newly formed woven bone and directly bridged by day 10 . Furthermore, the bone union in Group R was achieved mostly without a large external callus by day 14 . On the other hand, in Group F with the flexible plate, granulation tissue adjacent to the fracture site and anchoring callus associated with intramembranous ossification were apparent 7 days after the fracture. Cartilage tissue appeared around granulation tissue on day 7 , and bridging of the fracture site began through endochondral ossification. Finally, a large external callus was observed on day 21. Our results suggest that the 2 different MouseFix ${ }^{\text {TM }}$ plates activated two distinct fracture healing processes with different callus formation and cellular events. Many investigations based on clinical experience and experi- ments of rats, rabbits and other large animals have demonstrated these types of fracture healing $[9,23,25,26]$. However, the precise mechanism has not previously been fully elucidated.

Previous reports have revealed that several growth factors including bone morphogenetic proteins (BMPs), transforming growth factor (TGF)- $\beta$, insulin-like growth factors (IGFs) and FGFs are involved in regulating bone regeneration and remodeling during bone growth and repair $[1,5]$. However, no published studies have focused on differences in endogenous growth factor expressions in experimentally controlled mechanical environments. Fracture healing processes are not all the same. We speculated that differences in the mechanical environment would result in variable growth factor expressions, thereby leading to differences in healing processes. As described above, there were major differences in the early phase of endogenous FGF-2 expression between the two fracture healing processes. These results suggest that the production of FGFs at the fracture site plays an important role in fracture repair processes. Previous studies have shown that the effects of FGFs are exerted mainly on mesenchymal cell proliferation and that FGF-2 is a more potent mitogen for fibroblasts and pre-osteoblasts than for differentiated osteoblasts [20]. In addition, FGF- 2 has been shown to inhibit bone cell differentiation and matrix synthesis $[3,4,11,28]$. Thus, the stimulatory effect of FGF-2 on fracture repair appears to be due mainly to the stimulation of mesenchymal cell proliferation, and these cells then differentiate or support the differentiation of precursor cells. Compared to Group R with stable fixations, Group F had more interfragmentary movements that led to a difference in callus formation. The fracture callus was significantly larger in Group F than in Group R. In the acute phase of fracture healing, cellular proliferation in the inner layer of the periosteum is observed. Early cellular proliferation above the cortex appears to make a crucial difference in callus size. Several studies have demonstrated that mechanical forces stimulate the expressions of growth factors [7, 30]. Li et al. reported that FGF-2 synthesis results from an immediate-early gene induced by mechanical stress in osteogenic cells [17]. It is therefore possible that marked FGF-2 expression could be a direct consequence of a flexible environment. 
Using a fracture model of the rat femur with an intramedullary wire, Bolander et al. have studied the expressions of genes of endogenous growth factors during the initial injury response period $[2,12]$. These studies contributed basic data to research on the promotion of fracture healing processes by the application of exogenous growth factors. The application of exogenous FGF2 accelerated the healing of the bone fracture and segmental bone defect in animal models [13-15, 22, 27, 29]. Furthermore, recent evidences indicates that recombinant human FGF-2 hydrogel accelerates the healing of bone fractures in humans [16]. In the present study, we showed differences in endogenous FGF-2 expression between two fracture healing processes which were promoted by different mechanical environments. There is a wide range of variation in the fixation methods employed in clinical settings, and fracture healing processes are not homogeneous. The optimal timing of application may differ depending on the stability of the fracture site. The differences in endogenous growth factor expressions must be thoroughly understood to achieve the most effective application. Our findings provide information useful for promoting basic research on optimizing fracture healing processes utilizing exogenous FGF-2.

In conclusion, using a mouse fracture model, the expressions of endogenous growth factors were directly compared for the first time between different callus formations. There was a clear difference between the two different healing processes. The present results demonstrate that there are major differences in the early phase of endogenous FGF-2 expression between the two fracture healing processes associated with different internal fixators. We speculate that differences in the mechanical environment induce different growth factor expressions, thereby leading to differences in healing processes.

\section{Acknowledgments}

We would like to thank Synthes Japan Inc., for providing Mouse Fix ${ }^{\mathrm{TM}}$. Synthes Japan Inc. had no involvement in this study aside from providing Mouse Fix ${ }^{\mathrm{TM}}$. This work was supported in part by research grants from the Parents' Association of Kitasato University School of Medicine.

\section{References}

1. Barnes, G.L., Kostenuik, P.J., Gerstenfeld, L.C., and Einhorn, T.A. 1999. Growth factor regulation of fracture repair. $J$. Bone Miner. Res. 14: 1805-1815.

2. Bolander, M.E. 1992. Regulation of fracture repair by growth factors. Proc. Soc. Exp. Biol. Med. 200: 165-170.

3. Canalis, E., Centrella, M., and McCarthy, T. 1988. Effects of basic fibroblast growth factor on bone formation in vitro. J. Clin. Invest. 81: 1572-1577.

4. Canalis, E., McCarthy, T., and Centrella, M. 1988. Growth factors and the regulation of bone remodeling. J. Clin. Invest. 81: 277-281.

5. Canalis, E., McCarthy, T.L., and Centrella, M. 1991. Growth factors and cytokines in bone cell metabolism. Annu. Rev. Med. 42: 17-24.

6. Chao, E.Y., Aro, H.T., Lewallen, D.G., and Kelly, P.J. 1989. The effect of rigidity on fracture healing in external fixation. Clin. Orthop. Relat. Res. 241: 24-35.

7. Cillo, J.E. Jr., Gassner, R., Koepsel, R.R., and Buckley, M.J. 2000. Growth factor and cytokine gene expression in mechanically strained human osteoblast-like cells: implications for distraction osteogenesis. Oral Surg. Oral Med. Oral Pathol. Oral Radiol. Endod. 90: 147-154.

8. Claes, L.E. and Heigele, C.A. 1999. Magnitudes of local stress and strain along bony surfaces predict the course and type of fracture healing. J. Biomech. 32: 255-266.

9. Einhorn, T.A. 1998. The cell and molecular biology of fracture healing. Clin. Orthop. Relat. Res. 355: S7-21.

10. Grongroft, I., Heil, P., Matthys, R., Lezuo, P., Tami, A., Perren, S., Montavon, P., and Ito, K. 2009. Fixation compliance in a mouse osteotomy model induces two different processes of bone healing but does not lead to delayed union. J. Biomech. 42: 2089-2096.

11. Hurley, M.M., Kessler, M., Gronowicz, G., and Raisz, L.G. 1992. The interaction of heparin and basic fibroblast growth factor on collagen synthesis in 21-day fetal rat calvariae. Endocrinology 130: 2675-2682.

12. Jingushi, S., Heydemann, A., Kana, S.K., Macey, L.R., and Bolander, M.E. 1990. Acidic fibroblast growth factor (aFGF) injection stimulates cartilage enlargement and inhibits cartilage gene expression in rat fracture healing. J. Orthop. Res. 8: 364-371.

13. Kato, T., Kawaguchi, H., Hanada, K., Aoyama, I., Hiyama, Y., Nakamura, T., Kuzutani, K., Tamura, M., Kurokawa, T., and Nakamura, K. 1998. Single local injection of recombinant fibroblast growth factor-2 stimulates healing of segmental bone defects in rabbits. J. Orthop. Res. 16: 654-659.

14. Kawaguchi, H., Kurokawa, T., Hanada, K., Hiyama, Y., Tamura, M., Ogata, E., and Matsumoto, T. 1994. Stimulation of fracture repair by recombinant human basic fibroblast growth factor in normal and streptozotocin-diabetic rats. Endocrinology 135: 774-781.

15. Kawaguchi, H., Nakamura, K., Tabata, Y., Ikada, Y., Aoyama, I., Anzai, J., Nakamura, T., Hiyama, Y., and Tamura, M. 2001. Acceleration of fracture healing in nonhuman primates by fibroblast growth factor-2. J. Clin. Endocrinol. Metab. 
86: 875-880.

16. Kawaguchi, H., Oka, H., Jingushi, S., Izumi, T., Fukunaga, M., Sato, K., Matsushita, T., and Nakamura, K. 2010. A local application of recombinant human fibroblast growth factor-2 for tibial shaft fractures: a randomized, placebo-controlled trial. J. Bone Miner. Res. (in press).

17. Li, C.F. and Hughes-Fulford, M. 2006. Fibroblast growth factor- 2 is an immediate-early gene induced by mechanical stress in osteogenic cells. J. Bone Miner. Res. 21: 946955.

18. Matthys, R. and Perren, S.M. 2009. Internal fixator for use in the mouse. Injury 40: S103-109.

19. Mayahara, H., Ito, T., Nagai, H., Miyajima, H., Tsukuda, R., Taketomi, S., Mizoguchi, J., and Kato, K. 1993. In vivo stimulation of endosteal bone formation by basic fibroblast growth factor in rats. Growth Factors 9: 73-80.

20. McCarthy, T.L., Centrella, M., and Canalis, E. 1989. Effects of fibroblast growth factors on deoxyribonucleic acid and collagen synthesis in rat parietal bone cells. Endocrinology 125: 2118-2126.

21. Nakamura, K., Kawaguchi, H., Aoyama, I., Hanada, K., Hiyama, Y., Awa, T., Tamura, M., and Kurokawa, T. 1997. Stimulation of bone formation by intraosseous application of recombinant basic fibroblast growth factor in normal and ovariectomized rabbits. J. Orthop. Res. 15: 307-313.

22. Nakamura, T., Hara, Y., Tagawa, M., Tamura, M., Yuge, T., Fukuda, H., and Nigi, H. 1998. Recombinant human basic fibroblast growth factor accelerates fracture healing by enhancing callus remodeling in experimental dog tibial fracture. J. Bone Miner. Res. 13: 942-949.
23. Olerud, S. and Danckwardt-Lilliestrom, G. 1968. Fracture healing in compression osteosynthesis in the dog. J. Bone Joint Surg. Br. 50: 844-851.

24. Otto, T.E., Patka, P., and Haarman, H.J. 1995. Closed fracture healing: a rat model. Eur. Surg. Res. 27: 277-284.

25. Perren, S.M. 2008. Fracture healing. The evolution of our understanding. Acta Chir. Orthop. Traumatol. Cech. 75: 241-246.

26. Perren, S.M., Huggler, A., Russenberger, M., Allgower, M., Mathys, R., Schenk, R., Willenegger, H., and Muller, M.E. 1969. The reaction of cortical bone to compression. Acta Orthop. Scand. Suppl. 125: 19-29.

27. Radomsky, M.L., Aufdemorte, T.B., Swain, L.D., Fox, W.C., Spiro, R.C., and Poser, J.W. 1999. Novel formulation of fibroblast growth factor-2 in a hyaluronan gel accelerates fracture healing in nonhuman primates. J. Orthop. Res. 17: 607-614.

28. Rodan, S.B., Wesolowski, G., Yoon, K., and Rodan, G.A. 1989. Opposing effects of fibroblast growth factor and pertussis toxin on alkaline phosphatase, osteopontin, osteocalcin, and type I collagen mRNA levels in ROS 17/2.8 cells. J. Biol. Chem. 264: 19934-19941.

29. Tabata, Y., Yamada, K., Hong, L., Miyamoto, S., Hashimoto, N., and Ikada, Y. 1999. Skull bone regeneration in primates in response to basic fibroblast growth factor. J. Neurosurg. 91: 851-856.

30. Yeung, H.Y., Lee, S.K., Fung, K.P., and Leung, K.S. 2001. Expression of basic fibroblast growth factor during distraction osteogenesis. Clin. Orthop. Relat. Res. 219229. 